\title{
Reinterpreting the Einstein-Bergson Debate through Contemporary Neuroscience
}

\section{Abstract}

We aim to recast the famous debate between Albert Einstein and Henri Bergson in 1922 in the context of contemporary psychology and neuroscience as informed by phenomenological analysis. We show that their disagreement is not as deep as has been considered previously, including Einstein and Bergson themselves. We argue that Bergson's dynamic view of consciousness as time constituting complements embodied models of time consciousness in modern neurophenomenological approaches. The phenomenological unity of time and self, including its inherent dynamism, is similar to that of recent neurophysiological conceptualizations, which suggest that the constantly ongoing integration of somatic signals over time generates a floating experience of the emotional self across moments of self-awareness. Embodied concepts of consciousness are thus grounded in the dynamic processes constituting the living body. We highlight empirical evidence informed by recent neurophysiological studies showing that the experience of time and the explicit judgment of duration are governed by emotional and visceral processes which share a common underlying neural processing system, the interoceptive system. Especially the insular cortex has shown to be related to the judgment of time as dependent on bodily and emotional states.

\section{Introduction: the Debate between Einstein and Bergson}

The enormous cultural and scientific significance of the debate between Albert Einstein and Henri Bergson has been amply documented. ${ }^{1}$ As the scholarship on this momentous debate shows, academics and the public at large at the moment of the first encounter between Einstein and Bergson were quite divided, some firmly siding with Bergson against Einstein and vice versa. Einstein was probably quite concerned about the criticisms from Bergson, but eventually, Einstein

1 See Canales (2015); (2016).

2 OpenAccess. (c) 2022 Marc Wittmann, Carlos Montemayor, published by De Gruyter.

(cc) BY This work is licensed under the Creative Commons Attribution 4.0 International License. https://doi.org/10.1515/9783110753707-019 
gained the upper hand among both the scientific community and the public. To this day, the debate is considered as a clash between two towering figures with radically opposite views, and many would consider Bergson's perspective as either confused or not really in line with science.

Even at the time of their initial debate on April 6, 1922, and in spite of Bergson's reputation as one of the greatest philosophers at that time, ${ }^{2}$ Bergson faced harsh criticism as a confused and irrational thinker. ${ }^{3}$ This polarization of the debate quickly took it into dramatic proportions. Rationality and scientific methodology was associated with Einstein by his supporters, who thought of Bergson as a cult figure who promoted mystical approaches to the crucial topic of the nature of time. But Bergson's supporters thought of his criticism as an enormously important attack on a reductive and mechanistic perspective that deprived our view of the world of its natural vitality, turning it into a world of frozen relations-in the case of time, "a frozen grid that scientists call spacetime" (Montemayor 2013, xi), also known as the "block-universe".

Einstein famously took the passage of time to be a stubborn illusion. Bergson, by contrast, considered time as the foundation of vitality, or impulses, as well as of all dynamic and meaningful relations, including the sensorial properties we are conscious of. Is their debate, thus portrayed, between a view that presents reality as a set of frozen relations versus a view that reduces objective temporal properties to impulses and vital forces? A debate between the mechanistic agenda of scientific superiority and a view of the world based on the human spirit? On this superficial reading of the debate many distinctions would disappear from view; we would miss important nuances of how Einstein and Bergson clarified their views in interactions with other thinkers. In particular, we would ignore Bergson's constant interest in mathematics and physics as well as Einstein's interest in the question of what could explain the illusion of time.

Although there is some truth to the claim that this debate is a stark illustration of "a world largely split into science and the rest" (Canales 2015, 7), we believe contemporary neuroscience and current psychological approaches to phenomenology have much to offer to the project of recasting and reinterpreting this debate in new and insightful ways. It is not our purpose to settle the debate or claim a definitive interpretation of it. Our main claim is that if one understands Bergson as a phenomenologist with specific claims about the psychology of time, then the alleged chasm between Einstein's scientific side and Bergson's un-

2 See Dewey (1912).

3 See Berlin (1935); Russell (1912). 
scientific and irrational side starts to close and vanish. Isaiah Berlin gives us a clue as to how we can approach Bergson's perspective in his criticism of Bergson's work. Jimena Canales writes: "The historian and theorist Isaiah Berlin associated him [Bergson] with the 'abandonment of rigorous critical standards and the substitution in their place of casual emotional responses"' (Canales 2015, 13).

Surely, there is a place for the role emotion and consciousness play in our lives, and there are certainly scientific approaches to time consciousness. Bergson is not typically considered a phenomenologist, a lineage that is associated with Edmund Husserl and his students, who actually distanced themselves from Bergson (Canales 2015, 139-152). We briefly explore this issue in section 3, but it is important to appreciate that Bergson's emphasis on time as experienced through the vitality of emotions, motivations, and conscious awareness is a central topic of contemporary neuroscience. ${ }^{4}$ The main point of this discussion is to show that, given the current evidence on the issues Bergson's philosophy revolved around, his seemingly titanic disagreement with Einstein might be based on an unfortunate misunderstanding, given the contemporary perspectives on time in metaphysics and psychology. On the one hand, Einstein is right in insisting on the mind-independent character of temporal relations, as the next section shows. On the other hand, Bergson is also right in insisting on the importance of experienced time as essential to our understanding of ourselves, as section 3 shows. These are both plausible and even complementary perspectives on time. As mentioned, the key to appreciate how they might be complementary is to understand this debate in the light of contemporary neuroscientific approaches to the psychology and phenomenology of time. In other words, science has changed dramatically in the fields of cognitive psychology and neuroscience, providing empirical evidence that Einstein and Bergson did not have available. Recasting the debate between Einstein and Bergson under the light of contemporary psychology shows that their disagreement is not as deep as has been interpreted so far, and certainly not as deep as both Einstein and Bergson took it to be.

\section{Metaphysical Implications of the Einstein-Bergson Debate}

In what sense was Einstein right when he said that "the time of the philosophers did not exist" (Canales 2015, 5; Il n'y a donc pas un temps des philosophes). Dur-

4 See, e.g., Damasio (1999); Craig (2015). 
ing the event in which Einstein met Bergson, Einstein clarified what he meant by this. Canales reports: "What Einstein said next that evening was even more controversial: 'There remains only a psychological time that differs from the physicist's. At that very moment, Einstein laid down the gauntlet by considering as valid only two ways of understanding time: physical and psychological" (Canales 2015,5$)$. What kind of challenge is this? Can there really be a third time, besides the physical and the psychological?

Canales describes Bergson's response to this challenge as follows: "The simple, dualistic perspective on time advocated by Einstein appalled Bergson. The philosopher responded by writing a whole book dedicated to confronting Einstein. His theory is "a metaphysics grafted upon science, it is not science" (Bergson 1999, 47). The book in question, Duration and Simultaneity, lays out a conception of time that identifies time with the continuity of our inner life, not as a stage with subsequent and discrete parts or transitional snapshots, but as a vital flow that determines duration itself, in opposition to everything that is rigidly and metrically determined. Kant famously also identified time with the "inner sense" of our minds, and said that it is in this inner intuition that we arrive at our knowledge of arithmetic - as opposed to geometry, which is based on our intuition of space or "outer sense". Interestingly, Bergson believes this is a recapitulation of the scientific perspective Newton favored in terms of absolute time and space (Kant's purpose is to defend the Newtonian system). Thus, perhaps the best way to characterize Bergson's account of time as the vitally creative force of duration in "our lives" is to define it as a non-metric experience, or a conscious experience that cannot be reduced to any metric relation, such as simultaneity, temporal order, or "clock-based" duration. As discussed further below, this is an approach the phenomenological movement, notably Edmund Husserl, favored and elaborated on.

But surely metric relations matter to how our perception and experience of time are structured. Bergson and Einstein could not know all the details at the time of their debate, but cognitive scientists now have provided ample evidence showing that the circadian clock and a short "stop-watch" clock are very important to how we perceive time. Both clocks are "psychological" in Einstein's sense, because they help us represent environmental relations in terms of the temporal properties of events (see Montemayor 2013, for a philosophical account of how these clocks generate representations that ground justified beliefs about time). This is psychological time, but it fundamentally depends on the activity of clocks: neurally instantiated time-keepers. So metric relations matter, and must be somehow reconciled with the vital duration we experience with emotions.

What about the phenomenology of time, or what Bergson describes as the transcendental nature of the flow and unity of our experiences? It may be 
more productive to think of it, not as a third kind of time, what Einstein calls "the time of the philosophers". Instead, reflections about the phenomenology of time should be informed by our knowledge concerning physical and psychological time. The phenomenology of time cannot be completely independent from physical and psychological time and in that sense, a dualistic account is foundational in order to understand the flow of time. But also, the situation is not fully reductive -the non-metric character of the flow and phenomenology of time needs to be explained. Even if Einstein is right and the flow of time is illusory, we must explain what produces such an illusion.

A dual model of time perception explains some of the key features addressed by both Einstein and Bergson. Brief scales of time in the millisecond range are directly linked to temporal features of the environment but at the seconds' stage, the phenomenology of presence starts weaving all the contents of our conscious awareness in a unity that transcends the previous metric structures (Montemayor and Wittmann 2014). The experiential and visceral vitality of our emotions and agency, which are the central focus of Bergson's account of time, can be explained in terms of a dual model that distinguish agency from early sensorial temporal processing (Montemayor 2017a), unconscious processing from visceral and arousal-related temporal awareness (Montemayor 2017b) and with respect to the present, a phenomenal present from a sensorial present (Montemayor 2013). Early sensorial processing concerning the relations of time order and simultaneity may occur without conscious awareness at the first stage of processing (a pre-agential processing). Then the agent is equipped with the necessary representational framework for integrating arousal-related information with her overall conscious experience in the phenomenal present (distinct from the sensorial present that relates stimuli in terms of simultaneity). Thus, Bergson's account of time based on emotions and agency is compatible with the perception of metric properties, associated with time in physics (involving the relations of simultaneity, temporal order, and duration).

So, what exactly are the metaphysical implications of Einstein's challenge to Bergson? One can accept Einstein's characterization of a dualistic approach to time, as well as most of what he said about physical time in opposition to Bergson, partly based on the psychological findings and proposals just mentioned. In addition, as will be explained in the next section, one can still justify the importance of the phenomenological project formulated by Bergson. These are not antagonistic or irreconcilable tasks. From an epistemic point of view, our beliefs about temporal relation need to be justified and for this we need the environmentally-based metric relations of simultaneity, duration, and time order.

Bergson's characterization of Einstein's view as “metaphysics grafted upon science" cannot be right without some clarification. As Reichenbach (1958) ex- 
plains, it is because of Einstein's theory of Relativity that we have arrived at an empirically testable theory of time and space that is also susceptible of rigorous mathematical formalization, unlike all the preceding views on time and space which depended on axiomatic first principles and a priori intuitions. This is a complex issue ${ }^{5}$ and we will examine this claim from a phenomenological perspective in the next section. But the main point is that Einstein's contribution to the study of space and time was deeply philosophical. Reichenbach (1958) argues extensively for the case that Einstein transformed the epistemology of space and time by updating it with ideas that are equally applicable the psychological space and time as well as physical spacetime. Reichenbach shows that coordinative definitions are essential for the empirical, rather than strictly a priori, study of space and time and that these coordinative principles must be in place any time one asks the questions concerning what is the actual geometry of a space and time given conventions about rigid bodies - again, including psychological space and time (e.g., visual, auditory, proprioceptive). Bergson's general statement that Einstein's theory is "metaphysics grafted upon science" misses entirely this point.

If Einstein is right, and there are only physical and psychological time, what follows for the metaphysics of time? As mentioned, one immediate implication is the approach to this issue, shifting from exclusively a priori or armchair perspectives to a posteriori or empirical ones (Reichenbach takes this to be the most important contribution of Einstein, particularly against Kant). We offer in the concluding sections evidence for both psychological time and the phenomenology of time, as the latter functions as the informative basis of the former, thus confirming this implication. Crucially, all of the contemporary metaphysical views of time are attempts at addressing Einstein's theory. While Einstein seems to have favored a block view of the universe in which change or passage of time have no place and the present is not unique, many recent views of time modify the strict requirements of the block universe to accommodate either change or passage. ${ }^{6}$

For instance, scientific theories of static time similar to the block universe explain our experience of the passage of time by appealing to neuroscience, ${ }^{7}$ or by proposing that while events might be objectively static, change does occur in terms of temporal order in relation to the emergent macroscopic properties that we associate with psychological time. ${ }^{8}$ So-called "Growing Block" the-

5 See Ryckman (2005).

6 See contributions in Dolev and Roubach (2016).

7 See Barbour (1997).

8 See Rovelli (2018a); (2018b). 
ories appeal to real becoming, ${ }^{9}$ in a way that depends on a moment that can be characterized as the "now" involved in the passage of time. ${ }^{10}$ Arguably, any theory that appeals to a moment in which time branches, or in which there is a spotlight that demarcates the Growing Block of spacetime is presumably a view that depends on a kind of objective characterization of the present moment as uniquely relevant for demarcating the boundary between the real and the possible. ${ }^{11}$ However, there are convincing arguments showing that passage can be accommodated in a block universe view, without making our experience of passage fundamental or primitive. ${ }^{12}$ These are all metaphysical and scientific views of time that try to accommodate the kind of passage that seems to have been, at least partly, the focus of Bergson's theory of time. Contemporary versions of the A theory dispense with such a robust understanding of passage, for instance by emphasizing instead property instantiation. ${ }^{13}$ While there are intricate issues concerning the topology, metric, and other metaphysical aspects of time that are relevant for the precise formulation of the A and B theories, our focus here is on how Einstein and Bergson understood the physics of time in relation to psychology.

Bergson's charge against Einstein's theory, namely that it inadequately injects metaphysics into physics, has not aged well. Most contemporary philosophers and scientists find various philosophical insights in Einstein's theory, including unprecedented epistemological insights about the nature of spacetime. But the relation between the psychological and the phenomenological remains a crucial difficulty. Einstein might be right that there is no "third time" of the philosophers, but surely simply saying that there is psychological and physical time is not enough. The "stubborn illusion" of time frames our life very deeply, as Bergson described, and this phenomenon deserves careful explanation.

\section{Phenomenological Implications of the Einstein-Bergson Debate}

Many of the observations Bergson made in his original debate with Einstein and in subsequent exchanges are phenomenological. Interpreted as a phenomenolo-

\footnotetext{
9 See Dowker (2014).

10 See Smolin (2013); (2014); (2015).

11 See Zimmerman (2008).

12 See Skow (2015).

13 See Sullivan (2012).
} 
gist, Bergson presents a series of epistemic points that Einstein should have taken more seriously. In particular, Bergson's characterization of time as irreducible to discrete quantities or mechanical characterizations is one that plays a very important role in the reception of Einstein's theory within the physics community. These are issues concerning the conscious basis of metric relations, coordinate systems and mathematics itself. Experienced duration is a vital unity from which all these abstract characterizations of time emerge. This section illustrates how Bergson is not alone in making these points, and in fact, he is in illustrious scientific company. The concluding sections show how contemporary approaches to neuroscience and phenomenology can be used to update and reinterpret the Einstein and Bergson debate.

Hermann Weyl explicitly addressed the problem of "how to bridge the gap or 'chasm' between the intuitive continua of space and time and the mathematical continuum. Based on the work of Edmund Husserl, Weyl opted for phenomenal time as the source of our intuitions of the mathematical continuum" (Montemayor 2012, 56). Bergson's emphasis on the vital and life-dependent character of phenomenal time are even more in line with current scientific evidence, as the research on interoception discussed below demonstrates. But there is much in common between the Husserlian approach and Bergson's here. One can find some of the same themes, and criticism to the mechanical view of reality initiated by Husserl and later by Heidegger in the work of Bergson. In a discussion on the idea of duration contained in Time and Free Will (1889/1913), Bergson proposes that consciousness presents the sequence and homogeneity of numbers in a two-fold manner, as a discrete succession for cognition and as an underlying unit, the unit of conscious awareness. In the unit of phenomenal time, nothing is discrete and succession is replaced by seamless continuity. There is a very fundamental epistemic point about physics in these remarks, including the work of Einstein, as Weyl famously argued in various scientific works:

The epistemological thesis that cognition in the exact natural sciences is the joint ('relative') product of objective characterization in precise mathematical concepts, and the subjective 'immediate life of intuition', belongs to transcendental-phenomenological idealism. In its terms, the very sense of objectivity is constituted within 'transcendental subjectivity', and accordingly, there must indeed be a vestige within the 'objective world' represented by the mathematical/ conceptual theories of physics of its 'origin' in the 'absolute' being of the given-to consciousness. Through reflection on the applications of mathematics in physics, Weyl recognized an ineliminable trace of the subjective source of all objectivity in the arbitrary fixing of a local coordinate system (Ryckman 2005, 135).

The immediate life of intuition is grounded, according to Bergson, in the very vitality of emotion and creative feeling. In time, this insight became a cru- 
cial assumption of the view called "the Embodied Theory" of the mind. Ideas about embodiment also entered the debate surrounding how to best approach the phenomenological project originated by Husserl. Merleau-Ponty, in particular, explored the bodily-based forms of spatiality and temporality that were infused with expectation, emotion, and creativity, just the way Bergson envisioned (more on this below, particularly with respect to findings on interoception). On this Bergsonian account of the phenomenology of time, our experience of vitality in time, in our lives, is a source not only of creativity and visceral engagement, but also of attentive strategies towards formalizations that depend on the source of the flow of life-time for their content, such as numerical and geometric abstractions.

Thus, Einstein is partly right when he affirms there are only two times, psychological and physical. This does not mean, however, that he should have ignored Bergson's claims about the centrality of phenomenology. Weyl, who was as qualified a physicist as was Einstein, fully appreciated the importance of phenomenology, and he gave it, accordingly, a critical role in his scientific and philosophical writings. As Ryckman explains in the passage above, Weyl sees the inescapable appeal to coordinate systems as depending fundamentally on our subjective awareness of time. This obviously includes the kind of objective coordinative definition emphasized by Reichenbach in his work on Einstein.

Bergson, as a phenomenologist, can be vindicated in the tribunal of history. His role in his debate with Einstein was important for philosophical and scientific reasons, rather than ideological and mystical motives. But, to restate differently the previous point, what would it mean to say that the "time of our lives" associated with morality, free will and creativity is "not-psychological" and, even more puzzling, what would it mean for the time of our lives to be absolutely unrelated to metric relations, such as simultaneity and time order? The following sections address these two issues, first by giving an account of the perception of temporal relations and second, by providing evidence of the psychological and neural basis of the experience of time.

Bergson is right that the irreducible datum of time is the flow and passage of our inner lives, which manifests itself at its clearest in the visceral and empathic reactions we associate with the vitality of conscious awareness (see Montemayor and Haladjian 2015, for how these reactions are best understood as phenomenal consciousness, dissociated from the more strictly epistemic functions of attention). But this is not the whole story. This continuous flow is also the basis upon which more hypostatic forms of attention depend, particularly concerning mathematical objects. The foundation of coordinative systems for mathematics, physics, and science in general, can be found in this "immediate life of intuition". 
But what do these phenomenological observations have to do with what Einstein called "psychological time"? As explained above, with respect to the philosophy of space and time, Einstein's epistemological contribution was to move the debate from a strictly a priori analysis (judgments arrived at through reasoning and intuition alone) to a posteriori reasoning (judgments arrived at on the basis of empirical evidence). Perhaps a similar advancement may be necessary in the case of phenomenology and psychology of time. The next sections provide evidence that is relevant for this project; these are scientific findings that neither Einstein nor Bergson had available. Section 4 discusses evidence concerning the perceptual capacities underlying representations and inferences about metric structure, or the environmental properties of events that must be understood in terms of relations of simultaneity, duration, and temporal order. Section 5 discusses findings on the relevance of interoception for what Bergson called "lived time".

\section{Current State of Affairs in the Psychology and Neuroscience of Time Perception}

The systematic study of time perception became possible in the second half of the $20^{\text {th }}$ century with the advent and elaboration of internal-clock, or stopwatch, models developed in the cognitive sciences. ${ }^{14}$ In essence, these models posit that a pacemaker sends out pulses which are sent to and stored in an accumulator by passing through a gate. ${ }^{15}$ According to these models the number of pulses stored in working memory would represent experienced duration. Strong evidence for clock-like properties of this timing system, i.e., as counting of clock ticks, comes from empirical work showing how physiological manipulation of the assumed clock rate induces relative under- and overestimation in animals and humans (respective right- or leftward shifts in the psychometric response function). ${ }^{16}$

To more accurately account for human timing performance in a further developmental step, an element was added to the model pertaining to the attentional focus of a human observer. According to the attentional-gate model ${ }^{17}$ pulses are only registered when attention is directed to the passage of time and a

14 See Wearden (2016).

15 See Treisman (1963); (2011).

16 See Meck and Church (1983); Meck (1996).

17 See AGM; Zakay and Block (1997). 
switch is closed which in turn opens a gate to the accumulator. This means that the estimation of duration is always a dual task. An observer can divide attention between experienced time and non-temporal features of an event. During a period of waiting for a personally important event to occur (the phone call of a loved person) most of our attention is directed to the passing of time, which then leads to an overestimation of waiting time. ${ }^{18}$ According to the AGM, two factors modulate the estimation of duration: ${ }^{19}$ (1) An increased emotional (bodily) arousal level increases the pacemaker rate and in turn leads to relative greater accumulation of pulses during a given time span; (2) A stronger focus of attention to time similarly leads to more inflow of pulses in the accumulator as the postulated gate is more frequently open. Importantly, the AGM relates only to time judgments under prospective conditions in which subjects are explicitly instructed to attend to time. Under retrospective conditions, in contrast, study participants are unaware that they will later be asked to estimate time while they perceive an event. The task becomes retrospective when the participant is instructed to judge duration after the event has ended. Then memory load pertaining to that period of time defines subjective duration; the more recall of changing features of the past interval, the longer duration estimates. ${ }^{20}$

The AGM is used in purely behavioural studies to explain relative over- and underestimations in prospective time perception tasks in an intuitively plausible way. For example, concurrent secondary tasks lead to a relative underestimation of duration because attention is diverted from the primary timing task; with increasing work load of the secondary task an increasing underestimation of duration is detectable. ${ }^{21}$ In one study, less attractive faces were associated with a relative underestimation of duration as compared to neutral and attractive faces. ${ }^{22}$ This was interpreted as stemming from the dislike and therefore reduced attention to the unattractive stimuli. Regarding the factor of arousal, judging duration of emotional negative stimuli with presentation times around 2 seconds as compared to neutral stimuli in one study not only led to relatively greater physiological arousal (as measured with the skin conductance response) but the negative sounds also generated relatively longer subjective duration. ${ }^{23}$ Using video clips with presentation times between 16 and 40 seconds, slow-motion scenes, as compared to real-time scenes, induced lower arousal (lower respiration rate

18 See Jokic et al. (2018).

19 See Burle and Casini (2001); Droit, Volet and Meck (2007).

20 See Brown (1985); Zakay and Block (1997).

21 See Brown (1985).

22 See Ogden (2013).

23 See Mella et al. (2011). 
and smaller pupillary diameter) which in turn was associated with a systematic underestimation of duration. ${ }^{24}$ Although practically speaking empirical results do not always conform to the model's predictions, ${ }^{25}$ the AGM is popular because of its strong heuristic value in explaining everyday subjective time. In the case of experienced boredom, the focus of attention is predominantly on time, and duration expands. ${ }^{26}$ During entertaining activities, when one is absorbed in what is happening we do not attend to time and the event passes comparably quickly, time flies. ${ }^{27}$

Regarding the neural basis of subjective time, i.e., the question of how and where in the brain time in the range between milliseconds and multiple seconds is processed, for many decades research focused on several distinct neural systems. Researchers are informed about the implication of certain neural networks involved in duration judgment using neuroimaging techniques (fMRI, EEG), through neuropsychological testing of brain-injured patients, by the transient interruption of neural processes with transcranial magnetic stimulation (TMS), and by assessing the effects of psychopharmacological agents. For several decades two competing models were favored for explaining the neural basis for sensory and motor timing: (1) Fronto-striatal circuits comprising recurrent loops between frontal cortex (SMA), caudate-putamen, pallidum and thalamus, which are modulated by the dopamine system; ${ }^{28}(2)$ the cerebellum, which has non-overlapping neural modules with different neural delay properties which could encode duration. ${ }^{29}$ Empirical evidence also points to several other regions such as the right posterior parietal cortex, which might be involved in general magnitude processing of time, space, and number, ${ }^{30}$ and the insula, the interoceptive cortex, i.e., the primary cortical area for receiving bodily signals. ${ }^{31}$

Meta-analyses of functional neuroimaging studies shed light on the duration dependency of the neural correlates of time. It seems that although the identified brain areas are involved in both the processing of sub- and supra-second intervals, relative more activation of subcortical structures appears with sub-second stimuli, whereas relative more activation of cortical regions is detected with

24 See Wöllner et al. (2018).

25 See Jones (2019).

26 See Zakay (2015); Jokic et al. (2018).

27 See Wittmann (2015).

28 See Harrington et al. (2004); Hinton and Meck (2004).

29 See Ivry and Keele (1989).

30 See Bueti and Walsh (2009).

31 See Wittmann et al. (2010). 
supra-second stimuli. ${ }^{32}$ Such a duration-dependent distinction has also been detected for effects of manipulation on the serotonin and dopamine transmitter systems. For example, healthy participants in timing studies were genotyped to compare individual temporal processing performance with genetic polymorphisms. Whereas temporal discrimination variability of visual stimuli around $500 \mathrm{~ms}$ were associated with a polymorphism related to D2 receptor density in the striatum, timing variability with stimuli around $2 \mathrm{~s}$ was associated with a polymorphism related to synaptic dopaminergic metabolism in the prefrontal cortex. ${ }^{33}$ This duration-dependent distinction between striatum and prefrontal cortex fits with the above mentioned sub- and supra-second activation for subcortical and cortical brain regions, respectively. ${ }^{34}$ Gene polymorphisms related to the serotonin system, but not the dopamine system, were associated with duration discrimination of longer intervals, namely with several seconds duration (with an average of $4.8 \mathrm{~s}$ ). ${ }^{35}$ This conforms to behavioural findings of doubleblind, placebo-controlled, studies using microdoses of psychedelic substances, i.e., subthreshold doses without noticeable effects on experience, which act predominantly on the serotonergic system. Two studies using psilocybin ${ }^{36}$ and LSD $^{37}$ detected deviations of timing performance under the influence of microdoses only for intervals longer than 2 seconds. However, an entire cocktail of neurochemical systems contributes to the experience of time. The neurotransmitter GABA has been correlated with timing accuracy in the subsecond time range; ${ }^{38}$ the neuromodulator oxytocin induced a specific time dilation and compression effect for happy female and for happy male faces, respectively, in heterosexual male viewers. ${ }^{39}$

To sum up these elaborations, next to the above-mentioned involvement of several transmitter systems, multiple neural networks are involved in the processing of time on different time scales. ${ }^{40}$ Recent neuroimaging studies point to several areas in the frontal cortex (SMA, inferior frontal gyrus), right intraparitetal sulcus, the basal ganglia, and the insula. ${ }^{41}$ One could argue that starting in

32 See Nani et al. (2020).

33 See Wiener et al. (2011).

34 See Nani et al. (2020).

35 See Sysoeva et al. (2010).

36 See Wackermann et al. (2008).

37 See Yanakieva et al. (2019).

38 See Terhune et al. (2014).

39 See Colonnello et al. (2016).

40 For an extensive discussion of empirical findings, see Wittmann (2009), passim.

41 Nani et al. (2020); Teghil et al. (2019). 
the 1980ies researchers were trying to find a dedicated timing system, an internal clock in a neural network which would be solely responsible for the processing of duration. What has emerged over the last couple of years is the insight that there is not one mechanism or area in the brain responsible for encoding time, but that there is a manifold of brain functions which rely on general dynamic properties of neural circuits. ${ }^{42}$ From the distributed neural circuits with its intrinsic neural patterns of activity there might be one circuit that is predominantly involved according to modality, task requirements, and the duration of the interval processed. ${ }^{43}$ Alternatively, several of these neural circuits could work together in an integrated fashion to cover different functional subcomponents for tracking time. ${ }^{44}$ The heterogeneity of neural mechanisms and brain circuits explains why many patients with a variety of diagnosed neurological disorders are often affected in the accuracy and precision of duration judgment. In those patient groups performance rarely breaks down completely, e.g., in patients with essential tremor who are diagnosed with anatomically widespread cerebello-thalamocortical dysfunctions. ${ }^{45}$ If one neural system is damaged, other parallel systems can partially take over and compensate. Timed behaviour is still possible, albeit impaired.

\section{Interoception as the Basis for the Experience of Time}

In the following we focus on one functional and anatomical module of the brain related to interoceptive (bodily) awareness which, over the last few years, has been implicated in the processing of subjective time. We will discuss the connection between empirical results in the cognitive neurosciences on the relationship between time perception and interoception with phenomenologically inspired ideas of temporal experience. Starting with the systematic analysis of Maurice Merleau-Ponty, individual philosophers have held the idea that perception and action, including subjective time and motor timing, are embodied faculties of the mind. The further developments of the enactive/embodied cognition models of subjectivity accordingly claim that the phenomenal first-person perspective of

42 See Paton and Buonomano (2018).

43 See Buhusi et al. (2018); Issa et al. (2020).

44 See Tomasi et al. (2015).

45 See Pedrosa et al. (2016). 
experience and behaviour depends on the dynamics of the corporal self. ${ }^{46}$ A classic study was conducted by Martin Strack and his students (1988) which showed that feedback from facial muscles influence felt emotions: Participants rated cartoons as funnier while they were presented under the condition with a pen in the mouth that facilitated muscles expressing positive emotions (a smile) as compared to a condition where a pen inhibited smiling, i.e., a pen facilitated muscles expressing negative emotions.

Subjective feelings depend upon bodily signals, i.e., visceral and somatosensory feedback from the peripheral nervous system, which inform us about the state of the body. These somatic signals are integrated with sensory signals from the other senses and with reference to the motivational, social and cognitive situation; in sum this integration capacity leads to the conscious awareness of the momentary emotional condition of an individual. ${ }^{47}$ One neural processing route in the multistep integration of ascending somatic signals culminates in the insular cortex, where the primary entry location for the signals is the dorsal posterior insula. This brain region has been termed the primary interoceptive cortex, the primary area in the cortex to process signals from all bodily organs. ${ }^{48}$ The fact that the insula is richly connected with other brain areas (limbic, sensorymotor) substantiates the view that interoceptive (embodied) processes underlie perception, cognition, and emotion. Several studies have shown that the conscious awareness of visual stimuli depends on how close they occur to the heartbeat; the closer the external stimuli to the heart beat, the lower accuracy of perceptual detection. For example, a neuroimaging study showed that the neural signals from the heart, as recorded in the insula, suppressed visual information when it was presented too close to the heart beat. ${ }^{49}$ Cardiac timing effects were also shown with emotional stimuli. Fearful faces were judged as more emotionally arousing when photos were presented at the cardiac systole (the actual heart beat) compared to when they were presented at the diastole (the relaxation period between heart beats); these effects were correlated with activity in the amygdala and insula. ${ }^{50}$

The insular cortex contributes to the experience of duration in the sub-second and supra-second time domain as repeatedly documented in reviews of neuroimaging studies. ${ }^{51}$ In our own neuroimaging research we employed a duration

46 A prominent neurophenomenological approach is put forward by Varela et al. (1991|2016).

47 See Damasio (1999).

48 See Evrard (2019).

49 See Salomon et al. (2016).

50 See Garfinkel et al. (2014).

51 See Wiener et al. (2010); Teghil et al. (2019); Nani et al. (2020). 
reproduction task with multiple-second tone intervals (3, 9, 18 seconds) in which individuals are presented first with a tone of varying interval lengths (encoding phase) which subsequently have to be reproduced in duration (reproduction phase). ${ }^{52}$ Ramp-like increases in fMRI activation was detected in the insular cortex which corresponded to stimulus duration. Activity ended with the termination of the to-be-timed stimuli in both the encoding (dorsal posterior insula) and the reproduction period (anterior insula). The very same duration reproduction task also proved to be related to recorded body signals. Cardiac periods increased (the heart slowed down) and skin-conductance levels decreased (a sign of relaxation) progressively during encoding and the reproduction phases for auditory ${ }^{53}$ and visual intervals. ${ }^{54}$ In a similar duration reproduction task, but with much shorter acoustic and visual stimuli (500 to $1500 \mathrm{~ms}$ ), activity in mid-insula (encoding phase) and the left anterior insula (reproduction phase) was reported. ${ }^{55}$ The authors speculated that insula activity in this temporal task could be related to the feeling of time passage.

The feeling of time passing could be used in sentient beings as a way to track time. We will come back to this idea a little later. Recent work led by Alice Teghil convincingly points to the involvement of interoceptive processes in the perception of time. In their behavioural study two duration reproduction tasks with intervals lasting between 8 and 18 seconds were used, filled with (a) regular spaced and (b) with irregular spaced stimuli. ${ }^{56}$ The regular condition thus provided external cues on presented duration which the irregular task did not. Only timing accuracy of the duration reproduction task with irregular stimuli was predicted by trait-like interoceptive awareness assessed by the Self-Awareness Questionnaire. That is, the general ability to be more aware of somatic states helped subjects to be more accurate in time perception when no regular external signals were available; they consequently had to rely on the dynamics of their bodily self to judge duration. These findings are indicative of the assumption made above that different distributed timing systems might come into play with different weights, across individuals but also within a person depending on the task given. An inter-individual propensity to be more aware of bodily processes helped subjects to be more accurate in the timing task. With fewer external cues available subjects potentially had to rely more on internal signals. This interpretation is founded on a subsequent study by Alice Teghil and coworkers

52 See Wittmann et al. (2010), (2011).

53 See Meissner and Wittmann (2011).

54 See Otten et al. (2015).

55 See Bueti and Macaluso (2011).

56 See Teghil et al. (2020a). 
with the same duration reproduction task, where they showed that posterior insula connectivity, which was modulated by individual interoceptive awareness as measured with the Self-Awareness Questionnaire, correlated only with the irregular condition and not with the regular condition. ${ }^{57}$

In a different study using dynamic video clips with intervals between 1 and 64 seconds, duration estimates could be explained by the amount of changes in the contents shown in the scenes, and not by measured cardiac responses or eye recordings. ${ }^{58}$ Participants in that study might have used the accumulation of perceived events stored in working memory to deduce experienced duration. These studies in combination show that, depending on the outward structure of the changing world, we can use visual or (acoustic) cues to deduce time. Or, when these cues are absent or irregular, we can rely on our internal body changes to judge duration.

What happens when the insular cortex is neurologically damaged? In a single-case study, an epileptic patient who suffered from focal damage to the right anterior insula showed severe impairments in the timing of multiple-second time intervals in the above-mentioned duration reproduction task, an impairment that did not occur in epileptic patients with damage to other cortical regions. ${ }^{59}$ Relating to duration estimation of shorter intervals between 300 and $1500 \mathrm{~ms}$, one study recruited and tested twenty-one neurological patients with a stroke in either the right or left insular cortex and compared performance with that of twenty-one control subjects. ${ }^{60}$ Only patients with right-hemispheric lesions, and not those with left-hemispheric lesions, quite remarkably underestimated the presented durations.

A comprehensive account regarding the relationship between awareness of bodily states, emotions and subjective time has been provided by Bud Craig (2009; 2015). A link between the three objects of awareness (body, emotions, and time) was proposed because the experience of time is related to emotional and visceral processes which share a common underlying neural processing system, the interoceptive system which includes the insular cortex. Based on the constantly ongoing processing of ascending bodily signals from the periphery and the internal organs a series of conscious emotional moments is created over time. Our sense of the bodily and emotional self is informed and constantly updated through ascending somatic signals integrated in the insula (therefore Craig's programmatic book title "How do you feel?"). Accordingly, the sense of

57 See Teghil et al. (2020b).

58 See Suárez-Pinilla et al. (2019).

59 See Monfort et al. (2014).

60 See Mella et al. (2019). 
time passage would evolve by the awareness of successive moments of bodily and emotional self-realization within a social and cognitive context. In essence, Craig presents an embodied theory of consciousness as grounded in the dynamic processes constituting the living body.

\section{Phenomenological and Neurobiological Accounts of Temporality}

What we want to put forward in the context of this chapter is that Henri Bergson's dynamic view of consciousness as time constituting complements to some extent the embodied model of time consciousness we present here. We are not discussing Bergson's metaphysical accounts of time ${ }^{61}$, but-similar to Barry Dainton's (2017) analysis of Bergson-consider him as phenomenologist of time. According to phenomenological analyses in the $20^{\text {th }}$ century (Husserl, Heidegger, Merleau-Ponty), self-consciousness and time-consciousness are inseparable. Conscious awareness can be described as an island of presence in the continuous flow of what happens (Metzinger 2004). Conscious states are inherently given to me; phenomenal experience is mine (Nagel 1974). Time as continuous flow of events in the present moment is linked to someone who experiences something, i.e., conscious perception includes a basic form of selfconsciousness (Zahavi 2005). The phenomenologically deduced unity of time and self can be expressed as: "there is no time without a self; there is no self without time". ${ }^{62}$ Julian Kiverstein (2009) summarizes this unity within the framework of Edmund Husserl's conceptualization of the tripartite structure of temporal experience encompassing past, present, and future as follows: I become aware of what is happening now to me through what just happened to me and expectations of what might happen to me. Self-consciousness ("what happens to me") is created through the temporal structure of consciousness as constituted through what has just happened (retention) and what is about to happen (protention) in an extended present. ${ }^{63}$ This extended present which encompasses a past and future orientation however is dynamic as "...the new present is the passage from a future to the present and of the previous present to the past-time

61 For an attempt to link Bergson's metaphysical ideas of temporality with neurobiology, see Korf (2015).

62 "Time must be understood as a subject, and the subject must be understood as time" (Merleau-Ponty 2012, 483).

63 See Husserl (1928). 
itself in motion [...] within a single movement" (Merleau-Ponty 2012, 442). This description thus is not about a sequence of static moments, one after the other, but the moment itself is constituted by the movement of the tripartite structure of time. A phenomenologist would accordingly deny the notion of static present moments which could be added in succession along a mathematically thought line. The now, according to Heidegger (his summer lectures of 1927 -Heidegger 1975, 352), who explicitly refers to Bergson, has the character of transition, the now is not a point next to other points in time but it has an extension which is characterized by passage. Similarly, Henri Bergson states: "pure consciousness does not perceive time as a sum of units of duration [...] states of consciousness are processes and not things [...] in consequence it is impossible to cut off a moment from them without [...] altering their quality" (Bergson 1917, 196). Or, as Barry Dainton puts it: “... when we hear a sequence of notes unfolding as part of a melody, we not only hear each note seamlessly flowing into the next, but the individual notes are themselves inherently dynamic" (Dainton 2017, 95).

The phenomenological derivation of the unity of time consciousness and self-consciousness, including its inherent dynamism, is strikingly similar to the neurophysiological model by Bud Craig where the constantly ongoing integration of somatic signals over time generates a floating experience of the emotional self across moments of self-awareness. From a neurobiological perspective, the underlying neural machinery at work actually processes incoming bits of information in discrete steps as witnessed by brain oscillations with many different lower and higher frequencies. ${ }^{64}$ These neural oscillations underlie all cognitive functions including perceptual and motor timing. ${ }^{65}$ Multiple oscillatory neural activities are however organized and interwoven into an integrated spatio-temporal continuum of brain activity. One idea is that slow cortical potentials, spanning several seconds of duration, may generate the temporal width of experience, an extended present moment. ${ }^{66}$ The long phases of these low-frequency fluctuations would temporally integrate oscillatory neural activity from different brain regions in order to enable conscious awareness and the feeling of an extended "now". To juxtapose the phenomenological view and the neuroscientific hypothesis of an extended present, one could state that the underlying neural machinery generates the temporal platform of a subjective present (Pöppel 2009), an island of presence (Metzinger 2004). This extended present

64 See Buzsáki (2006).

65 See van Wassenhove (2016); Roehricht et al. (2018).

66 See Northoff (2014). 
moment constitutes the temporal frame within which the dynamic flow of perceived events happens over time leading to experienced temporality. ${ }^{67}$

In his analysis of Bergson's philosophy of temporality, Dainton (2017) sees strong evidence for an extensional account, although some passages across different works could be interpreted differently, i.e., favoring a retentional model. Dainton's elaboration of an extensional model suggests dynamic elements of experience within an extended moment and across (overlapping) extended moments which create the seamless continuity of experience. In contrasting inner duration from the time measured by clocks, Henri Bergson similarly writes in his Essaie sur les données immédiates da la conscience (Time and Free Will) that "... inner duration, perceived by consciousness, is nothing else but the melting of states of consciousness into one another" (Bergson 1913, 107). In Dainton's (2017, 101) parlance of this stream-like structure: "A is experienced as flowing into B, B is experienced as flowing into C, and C into D”.

According to Bergson experienced duration (la durée) is experiential time as an intensive non-quantifiable entity: "psychic states seem to be more or less intense [...] looked in their multiplicity, they enfold in time and constitute duration" (Bergson 1917, 224). That is pure experience of temporality: "the deepest conscious states have no relation to quantity, they are pure quality" (Bergson 1917, 137). At a later stage subjective time may be quantified, i.e., spatialized as "length of duration", by comparing psychic states with external events, i.e., defined by clocks for measuring objective time. But the pure impression of temporality is generated through the qualitative stream-like enfolding of inner states.

\section{Summary}

The unity of time and self-consciousness has an inherent temporal dynamism as described by phenomenologists over the last 130 years. These phenomenological descriptions are mirrored by neurophysiological conceptions, which postulate a similar interoceptive dynamism associated with our feeling of time and self. It is suggested that the constantly ongoing temporal integration of somatic signals in the brain generates a dynamic experience of the emotional self across moments of self-awareness. Accordingly, the conscious feelings of emotions, self-experience, and subjective time stem from the dynamism of the same neural system related to interoceptive processing. We highlighted specific empirical evidence

67 See Dorato and Wittmann (2020). 
informed by recent neurophysiological studies showing that the experience of time is governed by emotional and visceral processes which are based on the interoceptive processing system. Neuroimaging studies have repeatedly shown that the insular cortex is activated when individuals have to judge duration in the millisecond and second range. Peripheral-physiological indices such as the heartrate or breathing rate are related to the accuracy in perception of timed external events as well as to the judgment of duration. Subjective assessments of interoceptive awareness show how people who are more aware of their bodily condition are also more accurate in time perception. Henri Bergson is thereafter vindicated as he was right to emphasize the dynamism of the temporal self as becoming. Psychology and cognitive neuroscience have come to a similar, scientifically-inspired, conclusion. Seen under this perspective also Albert Einstein was right. Bergson's approach was actually psychological in the sense that psychology is informed by phenomenology.

\section{References}

Barbour, Julian (1997), Nows are all we need, in: Harald Atmanspacher/Eva Ruhnau (eds.), Time, temporality, now, Berlin.

Bergson, Henri (1913), Time \& Free Will, London.

Bergson, Henri (1999), Duration and Simultaneity, Manchester.

Berlin, Isaiah (1935), “Impressionist Philosophy”, in: London Mercury 32 (191), 489-490.

Brown, Scott (1985), "Time perception and attention: The effects of prospective versus retrospective paradigms and task demands on perceived duration”, in: Perception \& Psychophysics 38, 115-124.

Bueti, Domenica/Walsh, Vincent (2009), "The parietal cortex and the representation of time, space, number and other magnitudes", in: Philosophical Transactions of the Royal Society B 364, 1831-1840.

Bueti, Domenica/Macaluso, Emiliano (2011), "Physiological correlates of subjective time: evidence for the temporal accumulator hypothesis", in: Neurolmage 57, 1251-1263.

Buhusi, Catalin/Oprisan, Sorinel/Buhusi, Mona (2018), "Biological and cognitive frameworks for a mental timeline”, in: Frontiers in Neuroscience 12, 377-381.

Burle, Boris/Casini, Laurence (2001), "Dissociation between activation and attention effects in time estimation: implications for internal clock models", in: Journal of Experimental Psychology: Human Perception and Performance 27, 195-205.

Buzsáki, György (2006), Rhythms of the brain, Oxford.

Canales, Jimena (2015), The physicist and the philosopher: Einstein, Bergson, and the debate that changed our understanding of time, Princeton, New Jersey.

Canales, Jimena (2016), Einstein's Bergson problem: Communication, consensus, and good science, in: Yuval Dolev/Micheal Roubach (eds.), Cosmological and psychological time, Cham. 
Colonnello, Valentina/Domes, Gregor/Heinrichs, Markus (2016), "As time goes by: Oxytocin influences the subjective perception of time in a social context", in:

Psychoneuroendocrinology 68, 69-73.

Craig, (Bud) A.D. (2009), "Emotional moments across time: a possible neural basis for time perception in the anterior insula", in: Philosophical Transactions of the Royal Society $B$ 364, $1933-1942$.

Craig, (Bud) A.D. (2015), How do you feel? An interoceptive moment with your neurobiological self, Princeton, New Jersey.

Dainton, Barry (2017), “Bergson on temporal experience and Durée Réelle”, in: lan Phillips (ed.), The Routledge Handbook of Philosophy of Temporal Experience, London, 93-106.

Damasio, Antonio (1999), The feeling of what happens: body and emotion in the making of consciousness, San Diego.

Dewey, John (1912), “Preface” to A Contribution to a Bibliography of Henri Bergson, New York.

Dolev, Yuval/Roubach, Michael (2016) (eds.), “Cosmological and psychological time”, Cham.

Dorato, Mauro/Wittmann, Marc (2020), "The phenomenology and cognitive neuroscience of experienced temporality", in: Phenomenology and the Cognitive Sciences 19, 747-771.

Dowker, Fay (2014), "The birth of spacetime atoms as the passage of time", in: Annals of the New York Academy of Sciences 1326, $18-25$.

Evrard, Henry (2019), “The organization of the primate insular cortex", in: Frontiers in Neuroanatomy 13 (43), 1-21.

Garfinkel, Sarah/Minati, Ludovico/Gray, Marcus/Seth, Anil/Dolan, Raymond/Critchley, Hugo (2014), "Fear from the heart: sensitivity to fear stimuli depends on individual heartbeats", in: Journal of Neuroscience 34, 6573-6582.

Haladjian, Harry/Montemayor, Carlos (2015), “On the Evolution of Conscious Attention”, in: Psychonomic Bulletin and Review 22 (3), 595-613.

Harrington, Deborah/Boyd, Lara/Mayer, Andrew/Sheltraw, David/Lee, Roland/Huang, Mingxiong/Rao, Stephen (2004), "Neural representation of interval encoding and decision making”, in: Cognitive Brain Research 21, 193-205.

Heidegger, Martin (1975), Die Grundprobleme der Phänomenologie. Band 24. Gesamtausgabe. II. Abteilung: Vorlesungen 1923-1944, Frankfurt am Main.

Hinton, Sean/Meck, Warren (2004), "Frontal-striatal circuitry activated by human peak-interval timing in the supra-seconds range”, in: Cognitive Brain Research 21, 171-182.

Husserl, Edmund (1928), Vorlesungen zur Phänomenologie des inneren Zeitbewußtseins, Halle.

Issa, John/Tocker, Gilad/Hasselmo, Michael/Heys, James/Dombeck, Daniel (2020), "Navigating through time: A spatial navigation perspective on how the brain may encode time”, in: Annual Review of Neuroscience 43, 73-93.

Ivry, Richard/Keele, Steven (1989), "Timing functions of the cerebellum”, in: Journal of Cognitive Neuroscience 1, 136-152.

Jokic, Tijana/Zakay, Dan/Wittmann, Marc (2018), “Individual differences in self-rated impulsivity modulate the estimation of time in a real waiting situation”, in: Timing \& Time Perception 6, 71-89. 
Jones, Luke (2019), "The perception of duration and the judgment of the passage of time", in: Valtteri Arstila/Adrian Bardon/Sean Power/Argiro Vatakis (eds.), The Illusions of Time. Cham, Berlin, 53-67.

Kiverstein, Julian (2009), “The minimal sense of self, temporality and the brain”, in: Psyche $15,59-74$.

Korf, Jakob (2015), "Bergsonian theses in a contemporary (neuro-) biological context. Mind \& Matter 13, 105-128.

Meck, Warren (1996), “Neuropharmacology of timing and time perception”, in: Cognitive Brain Research 3, 227-242.

Meck, Warren/Church, Russell (1983), “A mode control model of counting and timing processes", in: Journal of Experimental Psychology: Animal Behavior and Processes 9, $320-334$.

Meissner, Karin/Wittmann, Marc (2011), "Body signals, cardiac awareness, and the perception of time”, in: Biological Psychology 86, 289-297.

Mella, Nathalie/Conty, Lawrence/Pouthas, Viviane (2011), "The role of physiological arousal in time perception: psychophysiological evidence from an emotion regulation paradigm", in: Brain and Cognition 75, 182-187.

Mella, Nathalie/Bourgeois, Alexia/Perren, Fabienne/Viaccoz, Aurélien/Kliegel, Matthias/Picard, Fabienne (2019), "Does the insula contribute to emotion-related distortion of time? A neuropsychological approach”, in: Human Brain Mapping 40, $1470-1479$.

Merleau-Ponty, Maurice (1945/2012), Phenomenology of Perception, New York.

Metzinger, Thomas (2004), Being no one. The self-model theory of subjectivity, Cambridge, MA.

Monfort, Vincent/Pfeuty, Micha/Klein, Madelyne/Collé, Steffie/Brissart, Hélène/Jonas, Jacques/Maillard, Louis (2014), "Distortion of time interval reproduction in an epileptic patient with a focal lesion in the right anterior insular/inferior frontal cortices”, in: Neuropsychologia 64, 184-194.

Montemayor, Carlos (2012), “Continuous and Discrete Time: Scientific Possibilities”, in: Kronoscope 12 (1), $52-72$.

Montemayor, Carlos (2013), Minding Time: A Philosophical and Theoretical Approach to the Psychology of Time, Leida.

Montemayor, Carlos/Wittmann, Marc (2014), “The Varieties of Presence: Hierarchical Levels of Temporal Integration”, in: Timing and Time Perception 2 (3), 325-338.

Montemayor, Carlos/Haladjian, Harry H. (2015), Consciousness, Attention, and Conscious Attention, Cambridge, MA.

Montemayor, Carlos (2017a), "Conscious awareness and time perception”, in: PsyCH Journal 6 (3), $228-238$.

Montemayor, Carlos (2017b), “Time perception and agency: A Dual Model”, in: Ian Phillips (ed.). The Routledge Handbook of Philosophy of Temporal Experience, New York, 201-212.

Nagel, Thomas (1974), “What it is like to be a bat?", in: The Philosophical Review 83, 435- 450 .

Nani, Andrea/Manuello, Jordi/Liloia, Donato/Duca, Sergio/Costa, Tomaso/Cauda, Franco (2019), "The Neural Correlates of Time: A Meta-analysis of Neuroimaging Studies", in: Journal of Cognitive Neuroscience 31, 1796-1826. 
Northoff, Georg (2014), "Slow cortical potentials and "width of present", in: Unlocking the brain Volume 2: Consciousness, Oxford.

Ogden, Ruth (2013), "The effect of facial attractiveness on temporal perception", in: Cognition \& Emotion 27, 1292-1304.

Otten, Simone/Schötz, Eva/Wittmann, Marc/Kohls, Niko/Schmidt, Stefan/Meissner, Karin (2015), "Psychophysiology of duration estimation in experienced mindfulness meditators and matched controls", in: Frontiers in Psychology 6 (1215).

Paton, Joseph/Buonomano, Dean (2018), "The neural basis of timing: distributed mechanisms for diverse functions", in: Neuron 98, 687-705.

Pedrosa, David/Nelles, Christian/Maier, Franziska/Eggers, Carsten/Burghaus, Lothar/Fink, Gereon/Wittmann, Marc/Timmermann, Lars (2016), "Variance of essential tremor patients' time reproduction deficits”, in: Movement Disorders 31, 1428-1429.

Pöppel, Ernst (2009), "Pre-semantically defined temporal windows for cognitive processing”, in: Philosophical Transactions of the Royal Society B: 364, 1887-1896.

Reichenbach, Hans (1958), The Philosophy of Space \& Time, New York.

Roehricht, Jonas/Jo, Han-Gue/Wittmann, Marc/Schmidt, Stefan (2018), "Exploring the maximum duration of the contingent negative variation", in: International Journal of Psychophysiology 128, 52-61.

Rovelli, Carlo (2018a), “Physics Needs Philosophy. Philosophy Needs Physics”, in: Foundations of Physics 48, 481-491.

Rovelli, Carlo (2018b), The order of time, New York.

Russell, Bertrand (1912), “The Philosophy of Bergson”, in: Monist 22, 321-347.

Ryckman, Thomas (2005), The Reign of Relativity: Philosophy in Physics 1915-1925, Oxford. Salomon, Roy/Ronchi, Roberta/Dönz, Jonathan/Bello-Ruiz, Javier/Herbelin, Bruno/Martet, Remi/Faivre, Nathan/Schaller, Karl/Blanke, Olaf (2016), "The insula mediates access to awareness of visual stimuli presented synchronously to the heartbeat", in: Journal of Neuroscience 36, 5115-5127.

Skow, Bradford (2015), Objective Becoming, Oxford.

Smolin, Lee (2013), Time Reborn, Boston.

Smolin, Lee (2014), “Time, laws and the future of cosmology”, in: Physics Today 67 (3), 38 doi: $10.1063 /$ PT.3.2310.

Smolin, Lee (2015), “Temporal naturalism”, in: Studies in History and Philosophy of Science Part B: Studies in History and Philosophy of Modern Physics 52, 86-102.

Strack, Fritz/Martin, Leonard/Stepper, Sabine (1988), “Inhibiting and facilitating conditions of the human smile: a nonobtrusive test of the facial feedback hypothesis", in: Journal of Personality and Social Psychology 54, 768-777.

Suárez-Pinilla, Marta/Nikiforou, Kyriacos/Fountas, Zafeirios/Seth, Anil/Roseboom, Warrick (2019), "Perceptual content, not physiological signals, determines perceived duration when viewing dynamic, natural scenes", in: Collabra Psychology 5, 1-16.

Sullivan, M. (2012), "The minimal A-theory" in: Philosophical Studies 158, 149-174.

Sysoeva, Olga/Tonevitsky, Alexander/Wackermann, Jiři (2010), "Genetic determinants of time perception mediated by the serotonergic system”, in: PLOS ONE 5, e12650.

Teghil, Alice/Boccia, Maddalena/D’Antonio, Fabrizia/Di Vita, Antonella/de Lena, Carlo/Guariglia, Cecilia (2019), “Neural substrates of internally-based and externally-cued timing: An activation likelihood estimation (ALE) meta-analysis of fMRI studies", in: Neuroscience \& Biobehavioral Reviews 96, 197-209. 
Teghil, Alice/Boccia, Maddalena/Nocera, Laura/Pietranelli, Veronica/Guariglia, Cecilia (2020a), "Interoceptive awareness selectively predicts timing accuracy in irregular contexts", in: Behavioural Brain Research 377, 112242.

Teghil, Alice/Di Vita, Antonella/D’Antonio, Fabrizia/Boccia, Maddalena (2020b). "Inter-individual differences in resting-state functional connectivity are linked to interval timing in irregular contexts", in: Cortex 128, 254-269.

Terhune, Devin/Russo, Sonia/Near, Jamie/Stagg, Charlotte/Kadosh, Roi (2014), “GABA predicts time perception", in: Journal of Neuroscience 34, 4364-4370.

Tomasi, Dardo/Wang, Gene-Jack/Studentsova, Yana/Volkow, Nora (2015), “Dissecting neural responses to temporal prediction, attention, and memory: effects of reward learning and interoception on time perception", in: Cerebral Cortex 25, 3856-3867.

Treisman, Michel (1963), "Temporal discrimination and the indifference interval: Implications for a model of the 'internal clock"”, in: Psychological Monographs: General and Applied $77,1-31$.

Treisman, Michel (2013), “The information-processing model of timing (Treisman, 1963): Its sources and further development”, in: Timing \& Time Perception 1, 131-158.

Van Wassenhove, Virginie (2016), “Temporal cognition and neural oscillations”, in: Current Opinion in Behavioral Sciences 8, 124-130.

Varela, Francisco/Thompson, Evan/Rosch, Eleanor (1991|2016), “The embodied mind: Cognitive science and human experience", Cambridge, MA.

Wackermann, Jiří/Wittmann, Marc/Hasler, Felix/Vollenweider, Franz (2008), "Effects of varied doses of psilocybin on time interval reproduction in human subjects", in: Neuroscience Letters 435, 51-55.

Wearden, John (2016), The psychology of time perception, London.

Wiener, Martin/Turkeltaub, Peter/Coslett, Branch (2010), “The image of time: a voxel-wise meta-analysis", in: Neurolmage 49, 1728-1740.

Wiener, Martin/Lohoff, Falk/Coslett, Branch (2011), “Double dissociation of dopamine genes and timing in humans", in: Journal of Cognitive Neuroscience 23, 2811-2821.

Wittmann, Marc (2009), “The inner experience of time”, in: Philosophical Transactions of the Royal Society B 364, 1955-1967.

Wittmann, Marc/Simmons, Alan/Aron, Jennifer/Paulus, Martin (2010), "Accumulation of neural activity in the posterior insula encodes the passage of time”, in: Neuropsychologia 48, 3110-3120.

Wittmann, Marc/Simmons, Alan/Flagan, Taru/Lane, Scott/Wackermann, Jiři/Paulus, Martin (2011), "Neural substrates of time perception and impulsivity", in: Brain Research 1406, 43-58.

Wittmann, Marc (2015), “Modulations of the experience of self and time”, in: Consciousness and Cognition 38, $172-181$.

Wöllner, Clemens/Hammerschmidt, David/Albrecht, Henning (2018), "Slow motion in films and video clips: Music influences perceived duration and emotion, autonomic physiological activation and pupillary responses", in: PloS ONE 13(6), e0199161.

Yanakieva, Steliana/Polychroni, Naya/Family, Neiloufar/Williams, Luke/Luke, David/Terhune, Devin (2019), "The effects of microdose LSD on time perception: a randomised, double-blind, placebo-controlled trial”, in: Psychopharmacology 236, 1159-1170.

Zahavi, Dan (2005), Subjectivity and selfhood: investigating the first-person perspective, Cambridge, MA. 
Zakay, Dan/Block, Richard (1997), “Temporal cognition”, in: Current Directions in Psychological Science 6, 12-16.

Zakay, Dan (2015), "Psychological time as information: The case of boredom”, in: Frontiers in Psychology 5 (917).

Zimmerman, Dean W. (2008), “The privileged present: Defending an A-theory of time”, in: Dean W. Zimmerman et al. (eds.), Contemporary Debates in Metaphysics, Malden, MA, $211-225$. 